\title{
Reduction and Evaluation of Elliptic Integrals ${ }^{1,2}$
}

\author{
By W. J. Nellis and B. C. Carlson
}

1. Introduction. To evaluate an elliptic integral, one reduces it (often by a substitution involving Jacobian elliptic functions) to a combination of three standard tabulated integrals, customarily Legendre's normal integrals of the first, second, and third kinds. A very long list of integrals with algebraic, trigonometric, or hyperbolic integrands has been compiled by Byrd and Friedman [1] and reduced to Legendre's integrals; shorter lists are given by Gröbner and Hofreiter [5] and MilneThomson [6].

The present paper proposes, and partially carries out, a scheme for reducing a large number of elliptic integrals by means of a relatively short list of formulas. The first step is to evaluate the integral at hand in terms of the hypergeometric $R$-function [2]; this step replaces transformation to an integrand containing Jacobian elliptic functions, which are entirely avoided in the present procedure. Partly because of the flexibility provided by the numerical parameters in the $R$-function, the first three formulas in Table I are able to serve the same purpose as some seven hundred of the formulas in [1]. The second step is to express the $R$-function (with specific values of the parameters) in terms of three standard $R$-functions which are similar to but somewhat different from Legendre's normal integrals. This step is accomplished by means of Table II and its eventual extensions, which provide a further economy of space relative to a table reducing integrals of Jacobian elliptic functions to Legendre's integrals.

It is the second step which at present limits the utility of this procedure to integrals which are not of the third kind. Although the standard $R$-functions of the first and second kinds, $R_{F}$ and $R_{G}$, have been described in some detail [3], the properties of a corresponding function of the third kind are still being investigated. Nevertheless, it seems useful at the present time to give an account of the procedure and to provide tables for reducing and evaluating integrals of the first two kinds.

Short numerical tables of the standard functions $R_{F}$ and $R_{G}$ are included to show their general behavior. More precise values may be computed by the algorithms in [4] or the program for automatic computation given in [7]. The arguments of the tables have been chosen with an eye to facilitating interpolation in eventual larger tables; in particular the region where interpolation becomes very difficult in Legendre's tables (near $\theta=\phi=90^{\circ}$ ) is now expanded into a strip extending to infinity. Before entering the tables it will usually be necessary in practice to compute two square roots, whereas two arc sines as well as two square roots are often needed to enter Legendre's tables.

\footnotetext{
${ }^{1}$ Based on the M.S. thesis of W. J. Nellis, Iowa State University, Ames, Iowa, March, 1965.

${ }^{2}$ Work supported by the Ames Laboratory of the U. S. Atomic Energy Commission, and by the National Aeronautics and Space Administration under Grant NsG-293 to Iowa State University.
}

Received July 2, 1965. 
TABLE I

(T.1) $\int_{x}^{y}(t-x)^{\alpha}(y-t)^{\beta}(p+q t)^{\gamma}(r+s t)^{\delta}(u+v t)^{\epsilon} d t$ $=(y-x)^{\alpha+\beta+1} B(\alpha+1, \beta+1)(p+q x)^{\gamma}(r+s x)^{\delta}(u+v x)^{\epsilon}$

$\cdot R\left(\alpha+1 ; \alpha+\beta+\gamma+\delta+\epsilon+2,-\gamma,-\delta,-\epsilon ; 1, \frac{p+q y}{p+q x}, \frac{r+s y}{r+s x}, \frac{u+v y}{u+v x}\right)$,

$(\operatorname{Re} \alpha>-1 ; \operatorname{Re} \beta>-1 ; x<y ;|\arg (p+q t)|<\pi$ for every $t$ in the interval $[x, y]$, and similarly for $r+s t$ and $u+v t$; by (3.3), $\alpha$ and $\beta$ may be interchanged on the right side if $x$ and $y$ are also interchanged on the right side except in the first factor).

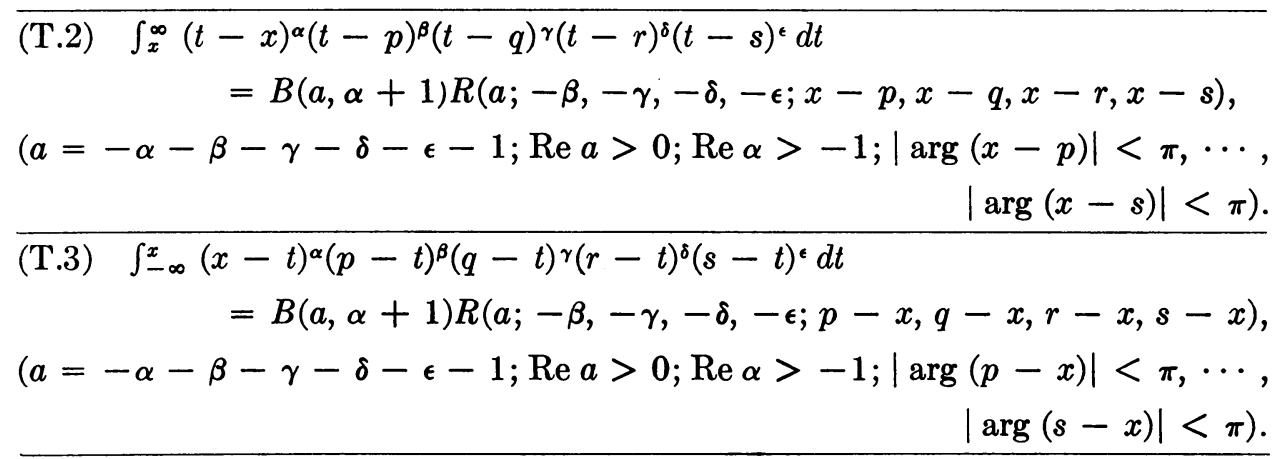

(T.4) If $0 \leqq \phi<\psi \leqq \pi / 2, f(\theta)$ may be $\sin \theta, \sin ^{2} \theta$, or $\cos ^{2} \theta$;

if $0 \leqq \phi<\psi \leqq \pi, f(\theta)$ may be $\cos \theta$;

if $0 \leqq \phi<\psi, f(\theta)$ may be $\sinh \theta, \cosh \theta, \sinh ^{2} \theta$, or $\cosh ^{2} \theta$ :

$$
\begin{aligned}
& \int_{\phi}^{\psi}|f(\theta)-f(\phi)|^{\alpha}|f(\psi)-f(\theta)|^{\beta}[p+q f(\theta)]^{\gamma}[r+s f(\theta)]^{\delta}[u+v f(\theta)]^{\epsilon}\left|\frac{d f}{d \theta}\right| d \theta \\
& =|f(\psi)-f(\phi)|^{\alpha+\beta+1} B(\alpha+1, \beta+1)[p+q f(\phi)]^{\gamma}[r+s f(\phi)]^{\delta}[u+v f(\phi)]^{\epsilon} \\
& \cdot R\left(\alpha+1 ; \alpha+\beta+\gamma+\delta+\epsilon+2,-\gamma,-\delta,-\epsilon ; 1, \frac{p+q f(\psi)}{p+q f(\phi)}, \frac{r+s f(\psi)}{r+s f(\phi)}, \frac{u+v f(\psi)}{u+v f(\phi)}\right),
\end{aligned}
$$

$(\operatorname{Re} \alpha>-1 ; \operatorname{Re} \beta>-1 ;|\arg [p+q f(\theta)]|<\pi$ for every $\theta$ in the interval $[\phi, \psi]$, and likewise for $r+s f$ and $u+v f$; by (3.3), $\alpha$ and $\beta$ may be interchanged on the right side if $\phi$ and $\psi$ are also interchanged on the right side).

2. List of Integrals. The hypergeometric $R$-function is defined for present purposes by the integral representation

$$
B(a, c-a) R\left(a ; b_{1}, \cdots, b_{n} ; z_{1}, \cdots, z_{n}\right)=\int_{0}^{\infty} t^{c-a-1} \prod_{i=1}^{n}\left(t+z_{i}\right)^{-b_{i}} d t,
$$

where $c \equiv \sum_{i=1}^{n} b_{i}$ and $B$ is the beta function. The path of integration is the positive $t$-axis; we assume $\left|\arg z_{i}\right|<\pi$ and take the principal value of $\left(t+z_{i}\right)^{-b_{i}}$ for all $i=1, \cdots, n$. Convergence of the integral requires $\operatorname{Re} c>\operatorname{Re} a>0$; when this condition is not satisfied, $R$ is defined by a hypergeometric series [2].

Several properties are evident from $(2.1): R$ is unchanged if the same permutation is applied to the parameters $(b)$ and to the arguments $(z)$; any $b$-parameter that is zero can simply be omitted along with the corresponding argument; $R$ is homoge- 
neous of degree $-a$ in $(z)$; and $R$ has the value unity when every one of its arguments is unity.

Elementary substitutions reduce the integrals of Table I to the form $(2.1)$ : let $t$ equal $(\tau x+y) /(\tau+1)$ in (T.1), $\tau+x$ in (T.2), and $x-\tau$ in (T.3). It is evident that (T.1) is related to (T.4) by $t=f(\theta)$, the absolute value signs being necessary if $f$ is a decreasing function of $\theta$.

3. Reduction to Standard Integrals. We choose as standard $R$-functions the normal integrals discussed in [3]:

$$
\begin{aligned}
& R_{F}(x, y, z)=R\left(\frac{1}{2} ; \frac{1}{2}, \frac{1}{2}, \frac{1}{2} ; x, y, z\right), \\
& R_{G}(x, y, z)=R\left(-\frac{1}{2} ; \frac{1}{2}, \frac{1}{2}, \frac{1}{2} ; x, y, z\right) .
\end{aligned}
$$

Both are completely symmetric in $x, y, z ; R_{F}$ is homogeneous of degree $-\frac{1}{2}$ and $R_{\sigma}$ of degree $+\frac{1}{2}$. Legendre's $F(\phi, k)$ can be expressed in terms of $R_{F}$ alone, while $E(\phi, k)$ is a combination of $R_{F}, R_{G}$, and an elementary function.

Let $2 b_{1}, 2 b_{2}, 2 b_{3}$ be odd integers. If $2 a$ is also an odd integer, the relations between associated $R$-functions [2] imply that

$$
C R\left(a ; b_{1}, b_{2}, b_{3} ; x, y, z\right)=C_{F} R_{F}(x, y, z)+C_{G} R_{G}(x, y, z)+C_{A}(x y z)^{-1 / 2},
$$

where the $C$ 's are homogeneous polynomials in $x, y, z$. A list of these polynomials is given in Table II. If $a$ is a negative integer, $R$ is itself a polynomial; if $a$ is a positive integer, one may use the table after applying the Euler transformation [2]:

$$
R\left(a ; b_{1}, b_{2}, b_{3} ; x, y, z\right)=x^{-b_{1}} y^{-b_{2}} z^{-b_{3}} R\left(a^{\prime} ; b_{1}, b_{2}, b_{3} ; x^{-1}, y^{-1}, z^{-1}\right),
$$

where $2 a^{\prime}$ is now an odd integer because of the definitions

$$
a^{\prime} \equiv c-a, \quad c \equiv b_{1}+b_{2}+b_{3} .
$$

The Euler transformation also explains the form of the third term on the right side of (3.2): since the $R$-function is unity when its first parameter vanishes [2], we have $R\left(\frac{3}{2} ; \frac{1}{2}, \frac{1}{2}, \frac{1}{2} ; x, y, z\right)=(x y z)^{-1 / 2}$.

Because the integrals of Table I necessarily lead to $R$-functions for which $a$ and $a^{\prime}$ have positive real parts, Table II has been restricted to cases in which $2 a$ is a positive odd integer and $a^{\prime}$ is a positive integer. Also, because the $R$-function is invariant under simultaneous permutation of $b$-parameters and arguments, we may assume $b_{1} \geqq b_{2} \geqq b_{3}$. These conditions being understood, Table II includes all cases in which the $b$-parameters are $\pm \frac{1}{2}$ or $\pm \frac{3}{2}$, as well as all cases in which one $b$-parameter is $\frac{5}{2}$ and the remaining two are $\pm \frac{1}{2}$.

Multiple integrals may lead to $R$-functions with $a$ or $a^{\prime}$ negative, $R_{G}$ itself being an example. Some integrals of this type are considered in [3], and some reduction formulas are given in [7]. The latter are useful also in extending Table II by means of recurrence relations [2, 7], of which we give only a single example since it is misprinted in [7]:

$$
\begin{aligned}
a(a+1) z_{1} z_{2} z_{3} R(a & +2)=a z_{1} z_{2} z_{3} \sum_{i=1}^{3}\left(1-a^{\prime}+b_{i}\right) z_{i}^{-1} R(a+1) \\
& +\left(a^{\prime}-1\right) \sum_{i=1}^{3}\left(a-b_{i}\right) z_{i} R(a)+a^{\prime}\left(a^{\prime}-1\right) R(a-1),
\end{aligned}
$$

where $R(a+m)$ stands for $R\left(a+m ; b_{1}, b_{2}, b_{3} ; z_{1}, z_{2}, z_{3}\right)$. 


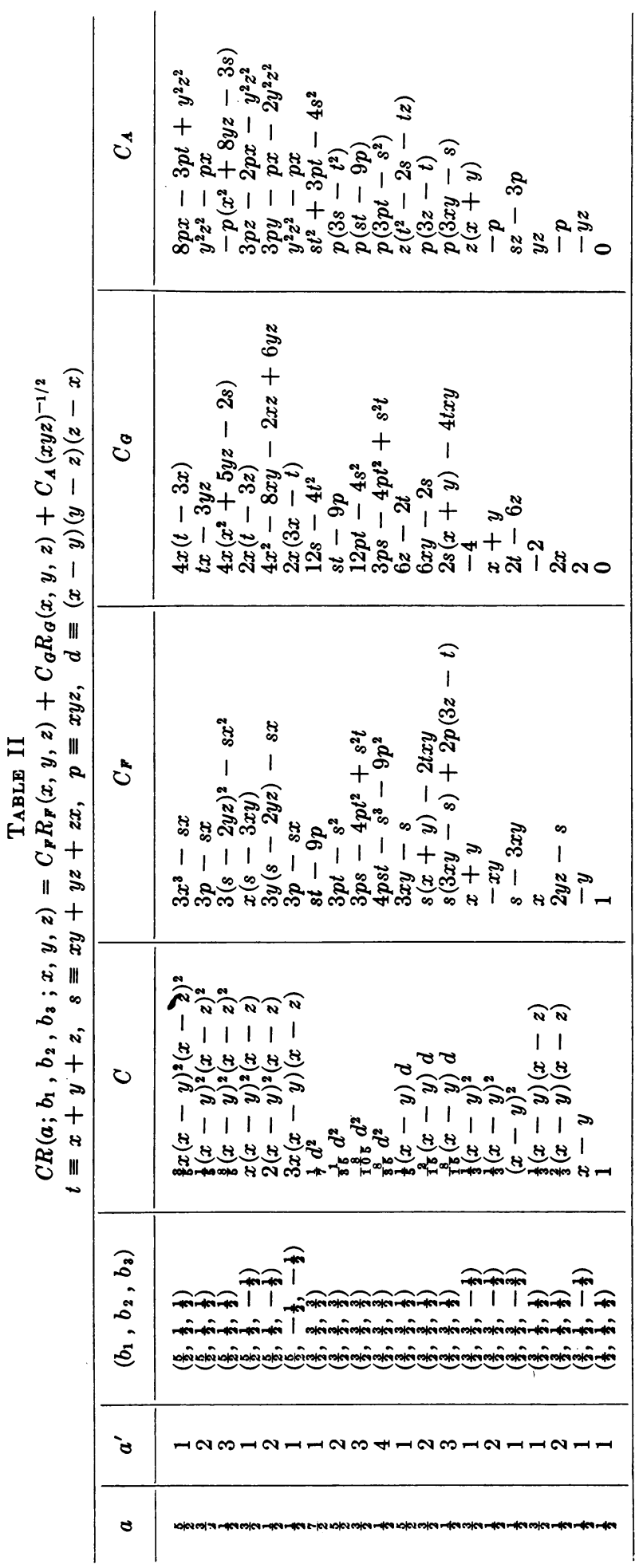


Although it is convenient to have a separate table of reduction formulas for complete elliptic integrals [7], they can alternatively be treated as incomplete integrals with one vanishing argument and reduced by using Table II. We suppose that an integral is evaluated by Table $\mathrm{I}$ in terms of an $R$-function with only two nonvanishing $b$-parameters. By (2.1) we may write

$$
\begin{aligned}
B\left(a, b_{1}+b_{2}-a\right) R(a ; & \left.b_{1}, b_{2} ; x, y\right) \\
& =B\left(a, b_{1}+b_{2}+b_{3}-a\right) R\left(a ; b_{1}, b_{2}, b_{3} ; x, y, 0\right)
\end{aligned}
$$

and choose $b_{3}$ to be $\frac{1}{2}$ or $\frac{3}{2}$. If $2 a, 2 b_{1}, 2 b_{2}$ are odd integers, Table II may be used to express the right side in terms of $R_{F, G}(x, y, 0)$; the polynomial $C_{A}$ will vanish with $z$.

The economy of Table II relative to a table of integrals of Jacobian elliptic functions is illustrated by the integrals of $c n^{2} u, n c^{2} u, d n^{2} u, n d^{2} u, c d^{2} u$, and $d c^{2} u$. All six integrals can be expressed by [2, Eq. (8.1)] in terms of $R\left(\frac{1}{2} ; \frac{3}{2}, \frac{1}{2},-\frac{1}{2} ; x, y, z\right)$; they differ from one another only by the six permutations of the arguments. Thus a single reduction formula for this $R$-function replaces six separate formulas reducing the integrals in question to Legendre's normal integrals.

4. Conjugate Complex Arguments. If a real integrand contains a half-odd-integral power of a polynomial with a pair of conjugate complex roots, reduction of the integrals by Tables I and II may lead to its expression in terms of $R_{F, G}(x, z, \bar{z})$, where $x$ is real and nonnegative and $\bar{z}$ denotes the complex conjugate of $z$. In order to replace the complex arguments by real nonnegative arguments, one may apply a Landen transformation [3]:

$$
\begin{aligned}
R_{F}(x, z, \bar{z}) & =R_{F}(u, v, w), \\
2 R_{G}(x, z, \bar{z}) & =4 R_{G}(u, v, w)-|z| R_{F}(u, v, w)-x^{1 / 2}
\end{aligned}
$$

where

$$
\begin{aligned}
& 2 u=|z|+\operatorname{Re} z, \\
& 2 v=|z|+x+|z-x|, \\
& 2 w=|z|+x-|z-x| .
\end{aligned}
$$

5. Numerical Tables. Because $R_{F, \sigma}(x, y, z)$ is homogeneous and symmetric, it suffices to tabulate $R_{F, G}(x, y, 1)$ in any one of the six regions into which the first quadrant of the $x, y$ plane is divided by the lines $x=1, y=1$, and $x=y$. For convenience of format we choose one of the two rectangular regions, say $0 \leqq x \leqq 1 \leqq y$. To avoid terms in $x^{1 / 2}$ as $x \rightarrow 0$, we tabulate $R_{F, G}\left(x^{2}, 1, y^{2}\right)$.

On the boundary of the region of tabulation, $R_{F, G}$ reduces to either an elementary function or a complete elliptic integral:

$$
\begin{aligned}
& R_{F}(1,1,1)=R_{G}(1,1,1)=1, \quad R_{F}(0,1,1)=2 R_{G}(0,1,1)=\pi / 2, \\
& R_{F}\left(x^{2}, 1,1\right)=2 R_{G}\left(x^{2}, 1,1\right)-x=\left(1-x^{2}\right)^{-1 / 2} \cos ^{-1} x, \\
& R_{F}\left(1,1, y^{2}\right)=2 R_{G}\left(1,1, y^{2}\right)-y=\left(y^{2}-1\right)^{-1 / 2} \cosh ^{-1} y, \\
& R_{F}\left(0,1, y^{2}\right)=y^{-1} K(k), \quad R_{G}\left(0,1, y^{2}\right)=\frac{1}{2} y E(k), \quad\left(k^{2}=1-y^{-2}\right) .
\end{aligned}
$$


The behavior of the functions as $y \rightarrow \infty$ can be found from the ascending Landen transformation [4]:

$$
\begin{aligned}
& R_{F}\left(x^{2}, 1, y^{2}\right)=\frac{1}{y} \ln \frac{4 y}{1+x}+O\left(\frac{\ln y}{y^{3}}\right), \\
& R_{G}\left(x^{2}, 1, y^{2}\right)=\frac{1}{2} y+O\left(\frac{\ln y}{y}\right) .
\end{aligned}
$$

More accurate asymptotic forms found by the same method can be modified by trial and error to get elementary functions which provide rough approximations to $R_{F, G}$ in the entire region of tabulation:

$$
\begin{aligned}
& f(x, y) \equiv\left(\frac{1}{y}+\frac{1+x+x^{2}}{7 y^{3}}\right) \ln \frac{4 y}{1+x}, \\
& g(x, y) \equiv \frac{1}{2} y-\frac{(1-x)^{2}}{8 y}+\frac{1+x^{2}}{4} f(x, y) .
\end{aligned}
$$

Defining correction factors $\varphi$ and $\gamma$ by

$$
\text { (5.4) } \quad R_{F}\left(x^{2}, 1, y^{2}\right)=\varphi(x, y) f(x, y), \quad R_{G}\left(x^{2}, 1, y^{2}\right)=\gamma(x, y) g(x, y) \text {, }
$$

\begin{tabular}{|c|c|c|c|c|c|c|c|c|c|c|c|}
\hline & \\
\hline & 0.0 & 0.1 & 0.2 & 0.3 & 0.4 & 0.5 & 0.6 & 0.7 & 0.8 & 0.9 & 1.0 \\
\hline $\begin{array}{l}1.0 \\
1.2 \\
1.4 \\
1.6 \\
1.8\end{array}$ & $\begin{array}{l}1.571 \\
1.431 \\
1.318 \\
1.225 \\
1.146\end{array}$ & $\begin{array}{l}1.478 \\
1.353 \\
1.251 \\
1.166 \\
1.094\end{array}$ & $\begin{array}{l}1.398 \\
1.285 \\
1.192 \\
1.114 \\
1.047\end{array}$ & $\begin{array}{l}1.327 \\
1.225 \\
1.140 \\
1.067 \\
1.005\end{array}$ & $\begin{array}{l}1.265 \\
1.171 \\
1.092 \\
1.025 \\
0.967\end{array}$ & $\begin{array}{l}1.209 \\
1.123 \\
1.050 \\
0.987 \\
0.933\end{array}$ & $\begin{array}{l}1.159 \\
1.079 \\
1.011 \\
0.952 \\
0.901\end{array}$ & $\begin{array}{l}1.114 \\
1.039 \\
0.975 \\
0.920 \\
0.872\end{array}$ & $\begin{array}{l}1.073 \\
1.003 \\
0.943 \\
0.891 \\
0.845\end{array}$ & $\begin{array}{l}1.035 \\
0.969 \\
0.913 \\
0.864 \\
0.820\end{array}$ & $\begin{array}{l}1.000 \\
0.938 \\
0.885 \\
0.838 \\
0.797\end{array}$ \\
\hline $\begin{array}{l}2.0 \\
2.2 \\
2.4 \\
2.6 \\
2.8\end{array}$ & $\begin{array}{l}1.078 \\
1.019 \\
0.967 \\
0.921 \\
0.880\end{array}$ & $\begin{array}{l}1.031 \\
0.976 \\
0.928 \\
0.885 \\
0.846\end{array}$ & $\begin{array}{l}0.989 \\
0.938 \\
0.893 \\
0.852 \\
0.816\end{array}$ & $\begin{array}{l}0.951 \\
0.903 \\
0.861 \\
0.822 \\
0.788\end{array}$ & $\begin{array}{l}0.916 \\
0.871 \\
0.831 \\
0.795 \\
0.763\end{array}$ & $\begin{array}{l}0.885 \\
0.842 \\
0.805 \\
0.770 \\
0.739\end{array}$ & $\begin{array}{l}0.856 \\
0.816 \\
0.780 \\
0.747 \\
0.718\end{array}$ & $\begin{array}{l}0.829 \\
0.791 \\
0.757 \\
0.726 \\
0.698\end{array}$ & & $\begin{array}{l}0.782 \\
0.747 \\
0.716 \\
0.688 \\
0.662\end{array}$ & $\begin{array}{l}0.760 \\
0.727 \\
0.698 \\
0.671 \\
0.646\end{array}$ \\
\hline $\begin{array}{l}3.0 \\
3.2 \\
3.4 \\
3.6 \\
3.8\end{array}$ & $\begin{array}{l}0.843 \\
0.809 \\
0.778 \\
0.750 \\
0.724\end{array}$ & $\begin{array}{l}0.780 \\
0.750 \\
0.724 \\
0.699\end{array}$ & $\begin{array}{l}0.753 \\
0.725 \\
0.700 \\
0.677\end{array}$ & $\begin{array}{l}0.728 \\
0.702 \\
0.678 \\
0.656\end{array}$ & $\begin{array}{l}0.733 \\
0.706 \\
0.681 \\
0.658 \\
0.637\end{array}$ & $\begin{array}{l}0.685 \\
0.662 \\
0.640 \\
0.620\end{array}$ & $\begin{array}{l}0.691 \\
0.666 \\
0.644 \\
0.623 \\
0.603\end{array}$ & $\begin{array}{l}0.672 \\
0.649 \\
0.627 \\
0.607 \\
0.588\end{array}$ & $\begin{array}{l}0.632 \\
0.611 \\
0.592 \\
0.574\end{array}$ & $\begin{array}{l}0.639 \\
0.617 \\
0.597 \\
0.578 \\
0.561\end{array}$ & $\begin{array}{l}0.623 \\
0.602 \\
0.583 \\
0.565 \\
0.548\end{array}$ \\
\hline $\begin{array}{l}4.0 \\
4.2 \\
4.4 \\
4.6 \\
4.8\end{array}$ & $\begin{array}{l}0.678 \\
0.657 \\
0.638 \\
0.620\end{array}$ & $\begin{array}{l}0.677 \\
0.655 \\
0.636 \\
0.617 \\
0.600\end{array}$ & $\begin{array}{l}0.655 \\
0.635 \\
0.616 \\
0.599 \\
0.582\end{array}$ & $\begin{array}{l}0.635 \\
0.616 \\
0.598 \\
0.582 \\
0.566\end{array}$ & $\begin{array}{l}0.617 \\
0.599 \\
0.582 \\
0.566 \\
0.551\end{array}$ & $\begin{array}{l}0.601 \\
0.583 \\
0.567 \\
0.551 \\
0.537\end{array}$ & $\begin{array}{l}0.585 \\
0.568 \\
0.553 \\
0.538 \\
0.524\end{array}$ & $\begin{array}{l}0.571 \\
0.555 \\
0.539 \\
0.525 \\
0.512\end{array}$ & $\begin{array}{l}0.542 \\
0.527 \\
0.513 \\
0.500\end{array}$ & $\begin{array}{l}0.545 \\
0.530 \\
0.515 \\
0.502 \\
0.490\end{array}$ & $\begin{array}{l}0.533 \\
0.518 \\
0.504 \\
0.492 \\
0.479\end{array}$ \\
\hline $\begin{array}{l}5.0 \\
5.2 \\
5.4 \\
5.6 \\
5.8\end{array}$ & $\begin{array}{l}0.603 \\
0.587 \\
0.572 \\
0.558 \\
0.545\end{array}$ & $\begin{array}{l}0.584 \\
0.569 \\
0.555 \\
0.541 \\
0.528\end{array}$ & $\begin{array}{l}0.539 \\
0.526 \\
0.514\end{array}$ & $\begin{array}{l}0.551 \\
0.537 \\
0.524 \\
0.512 \\
0.500\end{array}$ & $\begin{array}{l}0.523 \\
0.511 \\
0.499 \\
0.487\end{array}$ & $\begin{array}{l}0.523 \\
0.510 \\
0.498 \\
0.487 \\
0.476\end{array}$ & $\begin{array}{l}0.498 \\
0.486 \\
0.475 \\
0.465\end{array}$ & $\begin{array}{l}0.499 \\
0.487 \\
0.476 \\
0.465 \\
0.455\end{array}$ & $\begin{array}{l}0.476 \\
0.465 \\
0.455 \\
0.445\end{array}$ & $\begin{array}{l}0.478 \\
0.466 \\
0.456 \\
0.446 \\
0.436\end{array}$ & $\begin{array}{l}0.468 \\
0.457 \\
0.447 \\
0.437 \\
0.428\end{array}$ \\
\hline 6 & 32 & 0.516 & 0.502 & 0.489 & 0.477 & 0.465 & 0.455 & 0.445 & 0.436 & 0.427 & 0.419 \\
\hline
\end{tabular}

we have, for $0 \leqq x \leqq 1$,

TABLE IIIa

$R_{F}\left(x^{2}, 1, y^{2}\right)$ 
TABLE IIIb

$R_{G}\left(x^{2}, 1, y^{2}\right)$

\begin{tabular}{|c|c|c|c|c|c|c|c|c|c|c|c|}
\hline \multirow{2}{*}{$y$} & \multicolumn{11}{|c|}{$x$} \\
\hline & 0.0 & 0.1 & 0.2 & 0.3 & 0.4 & 0.5 & 0.6 & 0.7 & 0.8 & 0.9 & 1.0 \\
\hline & 785 & 0 & 79 & 814 & 2 & .855 & .880 & 07 & 36 & 77 & 1.000 \\
\hline & & & & & & 0.9 & & & & & \\
\hline 1. & & 0 . & 0.9 & 0.9 & 0. & 1.0 & 1. & & 4 & & 42 \\
\hline 1. & & 1. & 1.0 & 1. & 1.0 & 1.092 & 1.1 & 1.137 & 3 & 90 & 1.219 \\
\hline & & 125 & 1.1 & & 1.1 & 1.177 & 1.197 & 1.220 & 1.244 & 1.271 & 1.299 \\
\hline 2. & 1.211 & 1.214 & 1.221 & 1.232 & ; & 1. & 1. & 1.304 & 1.328 & 33 & 1.380 \\
\hline 2 & 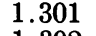 & 1.3 & 1.3 & 1.3 & 1.3 & 1.3 & 1. & 1. & 1. & & 64 \\
\hline 2. & & 1.39 & 1.401 & 1.411 & 1.4 & 1.440 & 1.458 & 1.478 & 1. & & 1.549 \\
\hline 2. & 1.4 & 1.48 & 1.493 & 1.502 & 1.515 & 1.530 & 1.547 & 1.567 & 1.588 & 1.611 & $\begin{array}{l}1.635 \\
\end{array}$ \\
\hline 2. & 1.5 & 1.5 & 1.585 & 1.5 & 1.6 & 1.621 & 1.638 & 1.656 & 1.677 & 1.699 & 1.723 \\
\hline 3. & 1.6 & 1.673 & 1.678 & & 1 & 1.7 & $1 . ?$ & 1.747 & 7 & U & 1.812 \\
\hline 3. & & 1. & 1. & & & & & & & & 1.901 \\
\hline 3. & & 1.8 & 1.866 & 1.8 & 1.8 & 1.8 & 1.913 & 1.931 & 1. & 1. & 1.992 \\
\hline 3. & 1.9 & 1.956 & 1.961 & 1.9 & 1.979 & 1.992 & 2.007 & 2.023 & 2.041 & 2.061 & 2.083 \\
\hline & & 2.051 & 2.056 & 2.063 & 2.073 & 2.086 & 2.100 & 2.116 & 2.134 & 2.153 & 2.174 \\
\hline 4. & 2 & & 2. & 2 & 2 & 2.1 & 2. & 2. & 2. & 2. & 2. \\
\hline 4 & & & & & & & & & & & 359 \\
\hline 4. & 2.337 & 2 . & 2.3 & 2.3 & 2.3 & 2.3 & 2.384 & 2. & 2.415 & 2. & 2.452 \\
\hline & & 2.4 & 2.4 & & & & & & & & 2.546 \\
\hline & 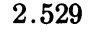 & 2.53 & 2.535 & 2.542 & 2.551 & 2.561 & 2.574 & 2.588 & 2.604 & 2.621 & 2.640 \\
\hline & 2 & 2 & 2 & 2 & & 2 & 2. & 2. & & & 2.734 \\
\hline & & & & & & & & & & & \\
\hline 5. & 2. & 2.8 & 2.8 & 2.8 & 2.8 & 2.8 & & & & & 2.923 \\
\hline & & 2.9 & 2.923 & & 2.9 & & & 2.971 & & & 3.01 \\
\hline & 015 & 3.016 & 3.020 & 3.026 & 3.034 & 3.043 & 3.055 & 3.067 & 3.082 & 3.097 & 3.114 \\
\hline 6.0 & 3.113 & 3.114 & 3.117 & 3.123 & 3.131 & 3.140 & 3.151 & 3.164 & 3.178 & 3.193 & $3: 209$ \\
\hline
\end{tabular}

$$
\begin{aligned}
& 0.98<\varphi(x, y)<1.01, \\
& (1 \leqq y \leqq 6), \\
& 0.999<\varphi(x, y)<1.001 \text {, } \\
& (6 \leqq y \leqq 25) \text {, } \\
& 0.9999<\varphi(x, y)<1.0001 \text {, } \\
& (25 \leqq y), \\
& 0.99<\gamma(x, y)<1.02 \text {, } \\
& (1 \leqq y \leqq 4) \text {, } \\
& 0.999<\gamma(x, y)<1.001 \text {, } \\
& (4 \leqq y \leqq 7) \text {, } \\
& 0.9999<\gamma(x, y)<1.0001 \text {, } \\
& (7 \leqq y) .
\end{aligned}
$$

The variables used in conventional tables of elliptic integrals are related to $x$ and $y$ by $1-k^{2} \sin ^{2} \phi=1 / y^{2}$ and $\cos \phi=x / y$; for example the last point in Legendre's table $\left(\phi=89^{\circ}=\sin ^{-1} k\right)$ corresponds approximately to $x=0.7, y=40$. That part of Legendre's table where interpolation is difficult $\left(\phi\right.$ and $\sin ^{-1} k$ both $\left.>85^{\circ}\right)$ is now expanded to occupy most of the strip $0 \leqq x \leqq 1, y>8$. Thus the present scheme of tabulation will facilitate interpolation when $k \sin \phi$ is close to unity.

Table III shows the behavior of $R_{F, G}\left(x^{2}, 1, y^{2}\right)$ to $3 \mathrm{D}$ accuracy in the region where the approximations (5.3) are inadequate for this purpose. Reference [7] contains a $4 \mathrm{D}$ table of $R_{F, \theta}(x, y, 1)$ for $x=0(0.05) 1, y=0(0.05) 1$. Algorithms for computing more precise values are given in [4], and a program for automatic computation (using the descending Gauss and ascending Landen transformations) can be found in 
[7]. This program was used to compute Table III on the IBM 7074 of the Iowa State University Computation Center.

6. Examples. The first two examples are taken from [6] and [1] to provide a direct comparison with familiar procedures. The third illustrates how to reduce a complete elliptic integral by Table II, and the fourth involves a polynomial with conjugate complex roots. The fifth is an example in which Table II can be used only after splitting the integral into two parts.

Example 1. Evaluate $I_{1}=\int_{2}^{4}\left[\left(x^{2}-2\right)\left(x^{2}-4\right)\right]^{-1 / 2} d x$. We put $x^{2}=t$ and use (T.1) to obtain

$$
\begin{aligned}
I_{1} & =\frac{1}{2} \int_{4}^{16}(t-4)^{-1 / 2}(t-2)^{-1 / 2} t^{-1 / 2} d t=\left(\frac{3}{2}\right)^{1 / 2} R\left(\frac{1}{2} ; \frac{1}{2}, \frac{1}{2}, \frac{1}{2} ; 1,7,4\right) \\
& =\left(\frac{3}{8}\right)^{1 / 2} R_{F}\left(\frac{1}{4}, 1, \frac{7}{4}\right) .
\end{aligned}
$$

By linear interpolation in Table IIIa with $x=0.5$ and $y=\frac{1}{2} \sqrt{ } 7=1.323$, we find $I_{1}=(0.612)(1.078)=0.660$. A more accurate value $[6, \mathrm{p} .603]$ is 0.65923 .

Example 2. Reduce to standard form the integral $I_{2}=\int_{0}^{\phi}(a+b \cos \theta)^{-1 / 2} d \theta$, where $a+b>0,0<\phi \leqq \pi, a+b \cos \phi \geqq 0$. We can either put $\cos \theta=t$ and use (T.1), or use (T.4) by writing

$$
\begin{aligned}
I_{2} & =\int_{0}^{\phi}(1-\cos \theta)^{-1 / 2}(1+\cos \theta)^{-1 / 2}(a+b \cos \theta)^{-1 / 2} \sin \theta d \theta \\
& =2(a+b)^{-1 / 2} \sin (\phi / 2) R_{F}\left(1, \frac{1+\cos \phi}{2}, \frac{a+b \cos \phi}{a+b}\right) .
\end{aligned}
$$

By using $F(\phi, k)=(\sin \phi) R_{F}\left(\cos ^{2} \phi, 1-k^{2} \sin ^{2} \phi, 1\right)$, one may verify agreement with the results in $[1$, p. 7] both for $a \geqq b>0$ and for $b \geqq a>0$; in the present notation there is no need to treat these cases separately.

Example 3. Evaluate $I_{3}=\int_{2}^{3}(t-2)^{1 / 2}(3-t)^{-1 / 2}(3 t-5)^{-3 / 2} d t$. From $(\mathrm{T} .1)$ and (3.6) we have

$$
I_{3}=B\left(\frac{3}{2}, \frac{1}{2}\right) R\left(\frac{3}{2} ; \frac{1}{2}, \frac{3}{2} ; 1,4\right)=B\left(\frac{3}{2}, 1\right) R\left(\frac{3}{2} ; \frac{3}{2}, \frac{1}{2}, \frac{1}{2} ; 4,1,0\right) .
$$

The last expression can be reduced by Table II :

$$
I_{3}=\frac{1}{3}\left[2 R_{F}(4,1,0)-R_{G}(4,1,0)\right] .
$$

Entering Table III with $x=0$ and $y=2$, we find

$$
I_{3}=\frac{1}{3}[2(1.078)-1.211]=0.315 \text {. }
$$

Example 4. Reduce $I_{4}=\int_{0}^{\sinh x}\left(t^{3}+t\right)^{-1 / 2} d t,(x>0)$. Using (T.1) we have $I_{4}=\int_{0}^{\sinh x} t^{-1 / 2}(t-i)^{-1 / 2}(t+i)^{-1 / 2} d t=2(\sinh x)^{1 / 2} R_{F}(1,1+i \sinh x, 1-i \sinh x)$. Eqs. (4.1) and (4.2) effect a transformation to real arguments:

$$
I_{4}=2(2 \sinh x)^{1 / 2} R_{F}\left(1+e^{-x}, 1+\cosh x, 1+e^{x}\right) .
$$

Since $(\sinh x) /(1+\cosh x)=\tanh (x / 2)$, we can use the homogeneity of $R_{F}$ to rewrite this in the form

$$
I_{4}=2\left(2 \tanh \frac{x}{2}\right)^{1 / 2} R_{F}\left(1-\tanh \frac{x}{2}, 1,1+\tanh \frac{x}{2}\right) .
$$


Example 5. Evaluate $I_{5}=\int_{0}^{24}[(t+1)(t+25)]^{-3 / 2}(4 t+1)^{1 / 2} d t$. Direct application of (T.1) leads to an $R$-function with $a=a^{\prime}=1$. In order to use Table II, we write $\int_{0}^{24}=\int_{0}^{\infty}-\int_{24}^{\infty}$ and apply (T.2):

$$
I_{5}=\frac{4}{3} R\left(\frac{3}{2} ; \frac{3}{2}, \frac{3}{2},-\frac{1}{2} ; 1,25, \frac{1}{4}\right)-\frac{4}{3} R\left(\frac{3}{2} ; \frac{3}{2}, \frac{3}{2},-\frac{1}{2} ; 25,49, \frac{97}{4}\right) .
$$

By Tables II and III the first term is

$$
\begin{aligned}
\frac{1}{144}\left[26 R_{F}\left(\frac{1}{4}, 1,25\right)-4 R_{G}\left(\frac{1}{4}, 1,25\right)+\frac{13}{5}\right] & \\
= & (0.00694)[26(0.523)-4(2.657)+2.6]=0.0387 .
\end{aligned}
$$

The second term is

$$
\begin{aligned}
\frac{1}{144}\left[74 R_{F}\left(25,49, \frac{97}{4}\right)-\right. & \left.4 R_{G}\left(25,49, \frac{97}{4}\right)+\frac{37}{35} \sqrt{97}\right] \\
& =\frac{1}{144}\left[\frac{74}{5} R_{F}(0.97,1,1.96)-20 R_{G}(0.97,1,1.96)+10.41\right] .
\end{aligned}
$$

By linear interpolation in Table III with $x=0.985$ and $y=1.4$, we have

$$
(0.00694)[(14.8)(0.889)-20(1.137)+10.41]=0.0057 .
$$

Thus $I_{5}=0.0387-0.0057=0.033$.

Iowa State University

Ames, Iowa

1. P. F. BYRD \& M. D. Friedman, Handbook of Elliptic Integrals for Engineers and Physicists, Die Grundlehren der mathematischen Wissenschaften, Bd. 67, Springer, Berlin, 1954. MR 15, 702.

2. B. C. CarLSON, "Lauricella's hypergeometric function $F_{D}$," J. Math. Anal. Appl., v. 7, 1963, pp. 452-470. MR 28 \#258.

3. B. C. Carlson, "Normal elliptic integrals of the first and second kinds," Duke Math. J., v. 31,1964 , pp. $405-419$. MR $29 \# 1360$.

4. B. C. CARLSON, "On computing elliptic integrals and functions," J. Math. and Phys., v. 44, 1965, pp. 36-51.

5. W. GRöBNER \& N. HofreITER, Integraltafel, 2nd ed., Springer, Vienna, 1958.

6. L. M. Mrne-Thomson, "Elliptic integrals," M. Ábramowitz \& I. Stegun (Eds.), Handbook of Mathematical Functions, National Bureau of Standards, Applied Mathematics Series, Vol. 55, U. S. Government Printing Office, Washington, D. C., 1964.

7. W. J. NeLus, Tables of elliptic integrals, M. S. thesis, Iowa State University, Ames, Iowa, 1965 . 